\title{
Clinical Evaluation of Glaucoma in Children
}

\author{
Nicholas G. Strouthidis • Maria Papadopoulos
}

Published online: 15 March 2013

(C) Springer Science + Business Media New York 2013

\begin{abstract}
Glaucoma in children is a potentially blinding condition. The suspicion of glaucoma in a child should be taken seriously and with a degree of urgency as early diagnosis and treatment can minimise visual loss. However, the clinical evaluation of children with glaucoma or suspected glaucoma can be challenging. Whilst a neonate may be examined in the clinic whilst sleeping or immediately following feeding, detailed examination to establish a diagnosis of glaucoma in infants or young children (or indeed to monitor once treatment has been instigated) may require examination under general anesthetic. Intraocular pressure (IOP) measurement needs particular care due to the variability of tonometry devices and the fact that IOP may be influenced by a number of factors. Optic disc assessment is critical in the diagnosis and monitoring of glaucoma in children and requires detailed dilated fundoscopic examination. Other examinations such as automated visual field testing and optic disc imaging, taken for granted in adult glaucoma patients, are highly dependent on patient cooperation. As such, they may not be possible in children until after the age of 6-7 years.
\end{abstract}

Keywords Pediatric glaucoma - IOP measurement . Optic disc $\cdot$ Primary congenital glaucoma

\section{Introduction}

Glaucoma in children is a rare condition characterized by the presence of elevated IOP and characteristic optic disc

N. G. Strouthidis $(\bowtie) \cdot$ M. Papadopoulos

Glaucoma Service, Moorfields Eye Hospital, NIHR Biomedical

Research Centre at Moorfields Eye Hospital NHS Foundation

Trust and UCL Institute of Ophthalmology, London, UK

e-mail: nick.strouthidis@moorfields.nhs.uk cupping. In addition to these features, glaucoma in infancy is associated with ocular enlargement, buphthalmos, which results from the biomechanical effects of elevated IOP in an eye with immature connective tissues (primarily collagen). The age of glaucoma onset determines the relevant questions to ask during the history taking and the clinical findings to identify. In other words, IOP measurement and optic disc appearance are fundamental features of the examination throughout the life a child with glaucoma, but in an infant whose eye is still vulnerable to the other effects of elevated IOP, proxies of elevated IOP (enlarged corneal diameter, increasing axial length and progressive myopia) need to be taken into consideration. In infants, the conclusion with regard to the diagnosis or progression of glaucoma must be based on the overall clinical findings and investigation results.

As a child grows older the cornea and sclera mature (become less elastic), so findings such as corneal diameter and axial length become less useful measurements. Improved cooperation with slit lamp examination allows more accurate assessment of IOP, and visualization of the optic disc and the nerve fibre layer with greater magnification. Hence, the child should be encouraged onto the slit lamp for evaluation when it appears this may be possible. Eventually, visual fields become feasible to assess functional vision, especially useful in advanced cases.

In this review we detail our approach to evaluating glaucoma in children. We also discuss some of the newer developments in the monitoring of childhood glaucomas, with particular reference to the measurement of IOP and posterior segment imaging.

\section{History and Presentation}

In a newborn or infant with glaucoma, the classic presenting triad is that of blepharospasm, photophobia and 
epiphora (without discharge). These symptoms are due to the presence of corneal edema from elevated IOP and may occur before the common signs of corneal haze and buphthalmos have manifested. Variability in the presentation of glaucoma in this age group relates to the magnitude of IOP rise. For example, a neonate with very high IOP at birth will present with cloudy, enlarged corneas. An infant with mildly elevated IOP is more likely to have an insidious presentation with buphthalmos but no photophobia or corneal clouding. Such cases may present later with progressive myopia or squint.

It is important to enquire about the age at onset of these symptoms and signs as it can influence the surgical prognosis of some conditions. For example, primary congenital glaucoma (PCG) presenting between 3 and 12 months of age is thought to respond better to angle surgery than that presenting at or soon after birth. In this age group it is also useful to ask about any problems during pregnancy such as maternal infections (particularly rubella) or forceps use during delivery, which may cause traumatic Descemet's breaks and thus be relevant to the differential diagnosis of glaucoma. In older children a history of trauma, intraocular inflammation, ocular surgery or corticosteroid use may be relevant.

A family history of childhood onset glaucoma and parental consanguinity are important to determine. If conditions associated with glaucoma have autosomal dominant inheritance or parental consanguinity exists, then siblings must also be examined for glaucoma. Furthermore, regardless of age, it is important to establish if there are any associated systemic problems that could result in an increased anesthetic risk and so influence your management.

Once the diagnosis is established, asking the parents of neonates and infants about changes in ocular appearance over time and in response to treatment can provide clues as to whether IOP control is satisfactory. Likewise, enquiring about changes in their child's visual behaviour or performance at school especially when associated with a change in visual acuity will also provide important information about glaucoma control.

\section{Examination}

The purpose of the clinical examination is either to make a diagnosis of glaucoma and establish the glaucoma subtype (vital in determining management), or in established glaucoma cases, to assess whether there has been progression. This examination is largely influenced by the child's ability to cooperate. Whereas a sleeping or feeding neonate can be examined in a clinic or office setting, most infants require examination under anesthesia (EUA) to be thoroughly assessed. In cases where doubt exists and a diagnosis or evidence of progression cannot be determined, then an EUA is advisable.

\section{General Appearance, Visual Behaviour and Visual Acuity Assessment}

Firstly, assess the child's general appearance and visual behaviour. Assessing visual acuity is important with regards amblyopia management and may be useful as a measure of progression in advanced cases. When a child is photophobic, reducing ambient lighting encourages he/she to open their eyes and allows better assessment for the presence of corneal oedema and buphthalmos.

\section{IOP Measurement}

Measuring the IOP is one of the key assessments, but can be difficult and challenging because it is potentially influenced by many factors such as: co-operation, eye movements, type of tonometer, anesthesia, intubation, speculum use and the state of the cornea (oedema, opacities and biomechanical properties). The IOP in normal children is lower than in adults and gradually increases with age to reach adult levels by the teenage years. The reference standard for IOP measurement remains Goldmann applanation tonometry (GAT), which is possible at the slit lamp in the majority of children by the age of 5 years and even earlier in exceptional circumstances. We consider the Perkins tonometer (a handheld version of GAT) to be the reference standard for use during EUA.

Other methods of IOP measurement, such as pneumatonometry, Tonopen ${ }^{\circledR}$ and iCare $^{\circledR}$ rebound tonometry are likely to give less accurate (albeit acceptable) approximations of the IOP. In the case of the Tonopen ${ }^{\circledR}$, this has been found to overestimate the IOP compared to the Perkins in both normal children and those with glaucoma $[1 \bullet, 2]$. The agreement between Tonopen ${ }^{\circledR}$ and GAT IOP measurements has recently been reported by the Pediatric Eye Disease Investigator Group, as an ancillary study of central corneal thickness (CCT) measurement in children [3]. In this large study, 439 normal, non-glaucoma subjects aged from birth to $<18$ years had IOP measured by Tonopen ${ }^{\circledR}$ and GAT at the slit lamp in the office setting (372 subjects) and by Tonopen ${ }^{\circledR}$ and by handheld GAT under general anesthetic (46 subjects). Mean differences and limits of agreement were found to vary as a function of IOP. Tonopen ${ }^{\circledR}$ measurements were found to be similar or slightly higher than GAT between 10 and $20 \mathrm{mmHg}$. As expected, thicker CCT led to higher IOP measurements using both devices. In normal children they found the mean differences between GAT and Tonopen ${ }^{\circledR}$ to be small but there is substantial testretest variability with Tonopen ${ }^{\circledR}$ and there is a larger difference between instruments in younger children. 
A drawback of both GAT and Tonopen ${ }^{\circledR}$ for neonates and infants in the office/clinic setting is the need for topical anesthesia. The iCare ${ }^{\circledR}$ rebound tonometer works by rebounding a probe off the surface of the cornea; IOP is estimated from the change in velocity of the probe as it rebounds. Its main advantage over other tonometers is that it does not require the instillation of topical anesthetic. Furthermore, it can be used in both the upright and supine positions (iCare ${ }^{\circledR}$ PRO) and is well tolerated $[4,5]$. As such, it is gaining popularity for pediatric IOP assessment in the office/clinic setting. However, a similar reliability to Tonopen ${ }^{\circledR}$ has been reported, with a tendency to overestimate IOP in glaucoma or suspected glaucoma cases as compared to applanation tonometry (Perkins and Goldmann) $[6,7]$.

We have recently assessed the agreement between IOP measurement by iCare ${ }^{\circledR}$ rebound tonometry and GAT in children with glaucoma between 5 and 16 years of age [8]. IOP measurements were acquired from 102 children (mean 11.9 years \pm 3.2 ) by two observers using rebound tonometry and then using GAT by a third observer. Intra- and inter-observer limits of agreement for rebound tonometry were $-2.7,3.0 \mathrm{mmHg}$ and $-5.8,6.0 \mathrm{mmHg}$ respectively. In general, iCare ${ }^{\circledR}$ rebound tonometry generated higher readings compared to GAT, with the magnitude of disagreement increasing with level of IOP and with higher CCT. Disagreements of greater than $10 \mathrm{mmHg}$ were not uncommon. Our interpretation of these results is that rebound tonometry agrees poorly with GAT in children with glaucoma, with frequent overestimation of IOP. However, measurements within the normal range are likely to be accurate. Therefore, a normal measurement of IOP by rebound tonometry could spare the child an unnecessary EUA. A high reading should alert the clinician to retest the IOP using applanation tonometry if possible and as usual to consider the IOP in the context of other clinical findings.

It should be noted that in the above study, $70 \%$ of children responded that they preferred rebound tonometry to applanation tonometry. The 'acceptability' and ease of use has led to its use as a domiciliary testing device. Investigators have shown that the iCare ${ }^{\circledR}$ can be used successfully at home by parents on their children with glaucoma [9]. The ability to test IOP by iCare ${ }^{\circledR}$ at home has led to the identification of similar diurnal fluctuations of IOP in normal children as are found in adults [10]. Fluctuations of $4-6 \mathrm{mmHg}$ have been identified, with higher readings in the early morning and lower readings in the late evening. The manufacturers of iCare ${ }^{\circledR}$ have now developed a device specifically for home use, the iCare $\mathrm{ONE}^{\circledR}$. This device is CE marked and is available commercially in Europe but is still awaiting FDA approval in the United States. This device differs from the standard iCare ${ }^{\circledR}$ in that it reports the IOP as a 'range' within which the mean of six IOP measurements lies. Readings are saved by date and time and can be downloaded onto a home computer using specialised software. In a recent study, IOP measurements using iCare $\mathrm{ONE}^{\circledR}$ and GAT were compared in 49 children with glaucoma and 11 glaucoma suspects (mean age 11.7 years, range 5-17 years), with measurements being undertaken both by a clinical examiner and by a parent [11•]. In all but one case, the parent could obtain a reliable measurement, with $98 \%$ of parents reporting the device to be easy to learn to use. The mean IOP difference between iCare $\mathrm{ONE}^{\circledR}$ and GAT was $3.3 \pm 4.0 \mathrm{mmHg}$ ( $p=0.001)$ when measured by the clinical examiner. The mean difference between iCare $\mathrm{ONE}^{\circledR}$ measurements between the clinical examiner and the parent was $1.9 \pm 1.9 \mathrm{mmHg}(p=0.008)$. iCare $\mathrm{ONE}^{\circledR}$ measurements taken by the clinical examiner were within $3 \mathrm{mmHg}$ of GAT measurements in $68 \%$ of cases. Of those with greater than $3 \mathrm{mmHg}$ difference, iCare $\mathrm{ONE}^{\circledR}$ measurements were higher than GAT measurements in $84 \%$.

Regardless of the technique for IOP measurement, certain principles should be considered. Ideally, the IOP should be taken with the eyes in the primary position and motionless in a relaxed child. Avoid measuring the IOP when using a lid speculum or when the child is crying or squeezing his/her lids as all of these may falsely elevate the IOP. IOP measurements must be obtained prior to pupil dilation as it can also affect the IOP. It is advisable to use the same type of tonometer for serial examinations as the literature suggests that there is less than perfect agreement between the various tonometers [3, 8]. Consider Goldmann application tonometry on the slit lamp as determined by the child's ability to cooperate. Finally, it is essential to consider IOP in the context of other clinical findings such as optic disc assessment and where relevant the ocular dimensions (corneal diameter, axial length), refraction and corneal clarity to determine whether glaucoma is present and/or whether it is progressing. If the IOP measurement is out of keeping with the other clinical findings, it may be inaccurate.

\section{IOP Measurement Under Anesthesia}

The main concern regarding IOP measurement during an EUA is that most general anesthetics cause a reduction of IOP [12]. The degree of IOP lowering is unpredictable and relates to factors such as the depth of anesthesia and its effect on cardiovascular tone $[12,13]$. Inhaled anesthetics may lower the IOP by $10-30 \mathrm{mmHg}$, which can have a profound effect on the diagnosis and management of these patients $[14,15]$. The finding of a normal IOP with the use of anesthetic agents known to reduce IOP does not exclude glaucoma. 
Although not universally practiced, it is our preference to use ketamine hydrochloride as IOP measurements are similar to those taken in awake infants [16••]. Ketamine hydrochloride is administered in our institution intravenously (following the use of local anesthetic patches) in conjunction with an agent that reduces bronchial secretions (atropine or glycopyrrolate) as well as midazolam which acts as a sedative and as an amnesic agent. Ketamine maintains airway tone and protective reflexes so intubation (which may result in increased IOP) is not usually required. Ketamine can cause a transient rise in IOP for a few minutes so the IOP should be measured several times in both eyes. If a surgical procedure needs to be undertaken at the end of the EUA, the anesthetist can switch to the standard preferred regime of inhaled and intravenous anesthesia. Other agents that similarly do not lower IOP are nitrous oxide and chloral hydrate [12, 17, 18].

Regardless of the anesthetic used it is important to have a consistent approach by using the same agent for serial examinations and to measure the IOP as soon as possible after initiation of general anesthesia (prior to intubation). This is followed by additional ocular examination (corneal diameter measurement, CCT measurement, anterior segment examination, gonioscopy, dilated indirect fundoscopy, cycloplegic refraction) and where indicated, general examination or venesection for genetic studies.

\section{Corneal Diameter Measurement}

During EUA the horizontal corneal diameter is measured from limbus to limbus with calipers and checked with a graduated ruler with estimation to the nearest $0.25 \mathrm{~mm}$. The normal neonatal horizontal corneal diameter is $10.5 \mathrm{~mm}$, increasing by about $1 \mathrm{~mm}$ in the first year of life. A diameter $>11 \mathrm{~mm}$ in a newborn and $12 \mathrm{~mm}$ in a child under the age of 1 year is highly suggestive of raised IOP, and with Haab striae is diagnostic. A measurement of $>13 \mathrm{~mm}$ in a child of any age is abnormal, as is marked asymmetry in corneal diameters. Corneal enlargement secondary to elevated IOP usually occurs before the age of 3 years, so it is an important marker to aid in establishing the diagnosis and in the monitoring of progression of glaucoma during infancy. For example, an increasing horizontal corneal diameter with successive longitudinal measurements suggests inadequate IOP control. In older children, measuring the corneal diameter may be relevant in making the correct diagnosis. For example, a case of PCG that spontaneously arrested in infancy and was undiagnosed, may present later as an older child with elevated IOP and glaucoma later. An increased corneal diameter indicates previously elevated IOP as an infant and a diagnosis of arrested PCG that has now relapsed.

\section{Central Corneal Thickness (CCT)}

Children with PCG, juvenile open angle glaucoma or of African heritage have thinner central corneas [19]. Corneal thickness is known to be thicker in patients with aniridia and aphakia. However, children with aphakia can develop glaucoma despite the theoretical overestimation of IOP that occurs in eyes with thick corneas [20]. A recent, small study of 40 eyes with PCG (mean age $13.6 \pm 4.8$ years) and 40 normal eyes (mean age $14.2 \pm 3.6$ years) suggested that there are altered corneal biomechanical responses in PCG [21]. There was a significant reduction in corneal hysteresis and corneal response factor in eyes with PCG as measured with the Reichert Ocular Response Analyzer ${ }^{\circledR}$, supporting the findings of an earlier similar study [22].

The definitive role for pachymetry in the evaluation of childhood glaucoma is currently uncertain. Although we continue to measure CCT in our clinics and during EUA, we do not advocate the use of correction factors or nomograms. Regardless of the CCT, the appearance of the optic disc remains the most important feature of the assessment.

\section{Anterior Segment Examination and Gonioscopy}

The anterior segment of a neonate or infant is examined with a hand held slit lamp in the clinic/office or during EUA and then using a table-mounted slit lamp once the child is old enough to cooperate, usually between 3 and 5 years of age. This is a very important part of the examination not only with regards the diagnosis of glaucoma but also in determining the type of glaucoma.

The cornea is examined for the presence of posterior embryotoxon, corneal edema, opacities and Haab striae. The use of an oblique slit beam and magnification can help identify subtle corneal features. Rarely, corneal dystrophies such as posterior polymorphous dystrophy can be diagnosed in neonates or young infants with corneal haze referred as glaucoma suspects, and should be considered especially when the corneal diameter is normal. The presence of corneal enlargement and Descemet's splits is indicative of previously elevated IOP during infancy. Persistent corneal edema may be a sign of poorly controlled glaucoma. Examining the iris and pupil for abnormalities is vital with regards to determining the type of glaucoma. Similarly, determining the coexistent presence of a cataract may influence the diagnosis and also affect the choice of surgical management.

Gonioscopy is a critical part of the examination in the assessment of a child with glaucoma just as it is in an adult, as it helps determine the glaucoma subtype. Information from gonioscopy often guides the choice of surgery. 
For example, subtle changes in the angle noted on gonioscopy may indicate that the child has Axenfeld Rieger Anomaly rather than PCG and therefore is likely to respond less well to angle surgery. In cases of uveitic glaucoma, extensive peripheral anterior synechiae identified on gonioscopy preclude angle surgery. Furthermore, when 'unilateral' PCG is diagnosed, the fellow eye often shows similar gonioscopic features along with a large corneal diameter and increased axial length but a normal optic disc. This fellow eye may have had significant postnatal angle maturation resulting in spontaneously arrested PCG. Detecting these changes is relevant, as these eyes need to be monitored closely as they can relapse at any stage. Therefore, gonioscopy should be performed at least once when possible. During EUA, direct gonioscopy can be achieved with a Koeppe lens in conjunction with a handheld slit lamp. The appearance of the angle can be documented photographically using the RETCAM ${ }^{\circledR}$ wide-angle lens. A four mirror Zeiss indirect goniolens is a good alternative during EUA or when examining a child on a slit lamp.

\section{Fundoscopy}

Evaluation of the appearance of the optic disc is absolutely critical and is perhaps the most sensitive indicator for glaucoma diagnosis and for the monitoring of progression. It is not influenced by other factors such as anesthesia or growth.

Dilated fundoscopy is performed once the IOP has been measured and anterior segment examination has been completed as it can spuriously elevate the IOP and alter the angle appearance. It is performed using an indirect ophthalmoscope with a 28D or 20D lens, a direct ophthalmoscope or using a condensing lens at the slit lamp when the patient is old enough to cooperate. In infants who dilate poorly due to aphakia or other reasons, the small pupil facility of the indirect ophthalmoscope is useful. It may not be possible to visualize the optic disc in infants with significant corneal edema or opacification. In these cases, ultrasound may be able to identify deep excavation, when present. The optic disc appearance, including its size, cup:disc ratio, focal areas of rim loss and nerve fibre layer defects should be carefully recorded with a drawing. Furthermore, in order to be able to assess optic disc change, it is important to have a representative image for baseline comparison. We advocate obtaining an optic disc image with photographs. At the time of EUA, this can be achieved with the RETCAM ${ }^{\circledR}$.

A cup:disc ratio of $>0.3$ in a Caucasian infant $<1$ year or $>0.5$ in an older child greatly raises the suspicion of glaucoma. Significant asymmetry of cupping when the optics discs are of equal size can also be suggestive of glaucoma. A documented increase in disc cupping is definitive evidence of inadequate IOP control and should lead to further intervention regardless of the magnitude of IOP.

Optic disc changes in children with glaucoma appear to be similar to those that occur in adults. However, children demonstrate the unusual feature of reversible disc cupping once the IOP is lowered providing it occurs before extensive damage has occurred. Younger children have a greater potential for reversal of disc cupping. This reversibility suggests that the high IOP has not led to loss of neural tissue or irreversible connective tissue remodeling as is observed in chronic adult high-tension glaucoma. More likely this represents a 'pre-laminar' phenomenon whereby the neural tissue is stretched but not lost, accompanied by reversible laminar bowing. Once normal IOP is established, the compression of the pre-laminar tissue is likely relieved and the lamina moves anteriorly, 'filling in' the cupping. A recent digital photographic analysis of eyes with pediatric glaucoma identified that a reduction in the size of the scleral canal accompanies the reversal of cupping [23]. Similar phenomenona have been demonstrated in vivo by OCT in adult eyes undergoing trabeculectomy [24, 25].

In addition to making a detailed assessment of the optic disc appearance another purpose of fundoscopy is to identify any relevant retinal features of other associated ophthalmic or systemic conditions, such as choroidal hemangioma in Sturge Weber syndrome or pigmentary retinopathy in congenital rubella syndrome.

\section{Refraction}

A reduction of hypermetropia or the presence of myopia in an infant is additional evidence of glaucoma. In addition, the observation of progressive myopia or a myopic shift in aphakic patients indicates inadequate IOP control. Cycloplegic refraction should be undertaken in the clinic, and if this is not possible retinoscopy can be performed during EUA. Once IOP has stabilised it is critical to identify and address ametropia and amblyopia in order to ensure optimal visual function.

\section{Ancillary Tests}

\section{Ultrasound}

Serial A-scans are useful to assess axial length whilst the scleral coat is still distensible (possibly until the age of 8 years). In glaucoma, axial length measurements tend to be asymmetrical as compared to normal eyes and eyes with megalocornea not related to IOP elevation. There is usually a decrease in axial length following successful IOP reduction. High resolution B-scan is useful to detect 
marked disc cupping in the presence of opaque ocular media or to help exclude posterior segment pathology such as choroidal haemangioma.

\section{Anterior Segment OCT and Ultrasound Biomicroscopy (UBM)}

These imaging modalities may be particularly useful in cases where dense corneal opacification prevents a view of intraocular structures, such as in Peters anomaly. Both devices are particularly useful for assessing the iridocorneal angle, to assess the iris conformation and insertion, as well as the presence of iris tumours/cysts. Of note, UBM requires the patient to be supine and utilizes a waterbath in contact with the eye. It is, therefore, only suitable for assessment of iridocorneal angle structure during an EUA except in much older children.

\section{Perimetry}

Static automated perimetry may be undertaken successfully by the age of 7-8 years. As with adults, there is a learning curve, with performance improving with repeated testing. It is important to consider only the 'raw' sensitivities and related grayscale as the mean defect, pattern standard deviation, pattern and total deviation plots are derived from age-matched data. The normative database for commercial automated perimeters currently do not include data acquired from child subjects. Once a visual field deficit is demonstrated, the visual field test should be repeated to confirm its presence. Furthermore, the visual field defect should correspond to the disc appearance in glaucoma. If such a correspondence is not present, then other causes of visual loss should be considered, which may be neurological in children with relevant systemic disease such as neurofibromatosis.

\section{Quantitative Optic Disc Imaging}

As with adult glaucoma, the exact role for semi-automated optic nerve head and retinal nerve fiber layer (RNFL) imaging devices in pediatric glaucoma has yet to be fully elucidated. In theory, once a child can be examined comfortably at the slit lamp then he/she should be able to undergo optic disc imaging (although attention and movement remain a problem as with perimetry). Currently, one of the issues preventing the adoption of imaging devices for pediatric glaucoma diagnosis is the fact that normative databases for the native classification algorithms do not include measurements obtained from children. This concern does not apply to the comparison of serial measurements, so these devices could be used for the monitoring of structural progression as in adults. A prospective, observational study in children with glaucoma confirmed that RNFL thickness and macular thickness measurements decline with increasing severity of glaucomatous disc damage as gauged by stereophotograph examination [26].

A recent study has attempted to establish the normal peripapillary RNFL thickness and macular thickness in 83 healthy children aged 5-15 years using the Spectralis ${ }^{\circledR}$ OCT device [27]. The mean RNFL thickness $(107.6 \pm 1.2 \mu \mathrm{m})$ was thicker than has been reported in adults. It is hoped that such studies will provide the necessary normative data to allow the newer generation of spectral domain OCT devices to become useful in assisting in the detection of glaucoma in children. Amongst the advantages of spectral domain OCT is that it has rapid image acquisition times, suggesting that it may be useful in younger children where attention and eye movements are problematic. Furthermore, these devices have improved axial depth penetration and resolution enabling structures deep to the surface of the cup to be visualized with greater clarity. This facility has enabled a group in Korea to exquisitely demonstrate progressive optic disc tilt and development of peripapillary atrophy in children with increasing myopic shift over time [28]. It is likely that these OCT imaging protocols will find a place in the assessment of glaucoma in children. Furthermore, hand held spectral domain OCT devices are now becoming available for use in the operating room which may further expand our ability to assess the optic disc in infants.

\section{Conclusions}

The evaluation of a child with glaucoma or suspected glaucoma can be challenging. If an examination in the clinic cannot definitively exclude glaucoma or glaucoma progression, then EUA is necessary. Intraocular pressure measurement can be influenced by a number of factors and so should not be the only method by which the presence or control of glaucoma is assessed. Use of the same tonometer and anesthetic for successive examinations is advisable. Optic disc assessment is critical in the diagnosis of and monitoring of glaucoma in children.

Acknowledgments The authors acknowledge a proportion of their financial support from the UK Department of Health through the award made by the National Institute for Heath Research to Moorfields Eye Hospital NHS Foundation Trust and UCL Institute of Ophthalmology for a Biomedical Research Centre for Ophthalmology. The views expressed in this publication are those of the authors and not necessarily those of the UK Department of Health.

Disclosure N.G. Strouthidis: has a grant/grant pending from American Health Assistance Foundation, has received payment for lecturing from MSD pharmaceuticals and Allergan, Inc, has received payment for manuscript preparation from Allergan, Inc. has received 
royalties from Elsevier and has received travel and accommodation expenses from Allergan, Inc.; M. Papadopoulos: none.

\section{References}

Papers of particular interest, published recently, have been highlighted as:

- Of importance,

-• Of major importance

1. - Levy J, Lifshitz T, Rosen S, Tessler Z, Biedner BZ. Is the tonopen accurate for measuring intraocular pressure in young children with congenital glaucoma? J AAPOS 2005;9:321-325. This study demonstrated that tono-pen IOP measurements disagree with Perkins applanation tonometry in children with primary congenital glaucoma presenting with IOP greater than $16 \mathrm{mmHg}$. In these cases, the tono-pen tended to overestimate IOP.

2. Garcia-Resua C, Gonzalez-Meijome JM, Gilino J, Yebra-Pimentel E. Accuracy of the new ICare rebound tonometer vs. other portable tonometers in healthy eyes. Optom Vis Sci. 2006;83: $102-7$.

3. Bradfield YS, Kaminski BM, Repka MX, et al. Comparison of Tono-Pen and Goldmann applanation tonometers for measurement of intraocular pressure in healthy children. J AAPOS. 2012;16:242-8.

4. Kageyama M, Hirooka K, Baba T, Shiraga F. Comparison of ICare rebound tonometer with noncontact tonometer in healthy children. J Glaucoma. 2011;20:63-6.

5. Lundvall A, Svedberg H, Chen E. Application of the ICare rebound tonometer in healthy infants. J Glaucoma. 2011;20:7-9.

6. Flemmons MS, Hsiao YC, Dzau J, Asrani S, Jones S, Freedman SF. Icare rebound tonometry in children with known and suspected glaucoma. J AAPOS. 2011;15:153-7.

7. Martinez-de-la-Casa JM, Garcia-Feijoo J, Saenz-Frances F, et al. Comparison of rebound tonometer and Goldmann handheld applanation tonometer in congenital glaucoma. J Glaucoma. 2009; 18:49-52.

8. Dahlmann-Noor AH, Puertas R, Tabasa-Lim S, et al. Handheld rebound tonometry in children with glaucoma. BMJ Open 2012 (in press).

9. Flemmons MS, Hsiao YC, Dzau J, Asrani S, Jones S, Freedman SF. Home tonometry for management of pediatric glaucoma. Am J Ophthalmol. 2011;152:470-482.e2.

10. Hsiao YC, Dzau JR, Flemmons MS, Asrani S, Jones S, Freedman SF. Home assessment of diurnal intraocular pressure in healthy children using the Icare rebound tonometer. J AAPOS. 2012;16: $58-60$.

11. - Gandhi NG, Prakalapakorn SG, El-Dairi MA, Jones SK, Freedman SF. Icare ONE Rebound Versus Goldmann Applanation Tonometry in Children With Known or Suspected Glaucoma. Am J Ophthalmol. 2012;154:843-849 e1. In this study, IOP measurements by GAT were compared with Icare ONE measurements acquired by clinic examiner and by the child's parent/guardian. IOP measurement with Icare ONE was found to be accurate and well tolerated and therefore may be suitable for home monitoring.
12. Self WG, Ellis PP. The effect of general anesthetic agents on intraocular pressure. Surv Ophthalmol. 1977;21:494-500.

13. Hetherington J Jr, Shaffer RN. Tonometry and tonography in congenital glaucoma. Invest Ophthalmol. 1968;7:134-7.

14. Quigley HA. Childhood glaucoma: results with trabeculotomy and study of reversible cupping. Ophthalmology. 1982;89: 219-26.

15. Ausinsch B, Munson ES, Levy NS. Intraocular pressures in children with glaucoma during halothane anesthesia. Ann Ophthalmol. 1977;9:1391-4.

16. •• Blumberg D, Congdon N, Jampel H, et al. The effects of sevoflurane and ketamine on intraocular pressure in children during examination under anesthesia. Am $J$ Ophthalmol. 2007;143:494-499. In this randomized clinical trial, IOP measurement was compared in children undergoing examination under either inhaled sevoflurane or intramuscular ketamine. IOP measurements were found to be significantly lower in the sevoflurane group and it was suggested that IOP measured after ketamine sedation more likely reflects the awake IOP.

17. Lalwani K, Fox EB, Fu R, Edmunds B, Kelly LD. The effect of nitrous oxide on intra-ocular pressure in healthy adults. Anaesthesia. 2012;67:256-60.

18. Fox BE, O'Brien CO, Kangas KJ, Murphree AL, Wright KW. Use of high dose chloral hydrate for ophthalmic exams in children: a retrospective review of 302 cases. J Pediatr Ophthalmol Strabismus. 1990;27:242-4.

19. Rushood AA, Zahrani MH, Khamis A. Central corneal thickness in full-term Saudi newborns. Acta Ophthalmol. 2012;90:e355-8.

20. Chen TC, Walton DS, Bhatia LS. Aphakic glaucoma after congenital cataract surgery. Arch Ophthalmol. 2004;122:1819-25.

21. Gatzioufas Z, Labiris G, Stachs O, et al. Biomechanical profile of the cornea in primary congenital glaucoma. Acta Ophthalmol. 2013;91:e29-34.

22. Kirwan C, O'Keefe M, Lanigan B. Corneal hysteresis and intraocular pressure measurement in children using the reichert ocular response analyzer. Am J Ophthalmol. 2006;142:990-2.

23. Mochizuki H, Lesley AG, Brandt JD. Shrinkage of the scleral canal during cupping reversal in children. Ophthalmology. 2011; 118:2008-13.

24. Lee EJ, Kim TW, Weinreb RN. Reversal of lamina cribrosa displacement and thickness after trabeculectomy in glaucoma. Ophthalmology. 2012;119:1359-66.

25. Reis AS, O'Leary N, Stanfield MJ, Shuba LM, Nicolela MT, Chauhan BC. Laminar displacement and prelaminar tissue thickness change after glaucoma surgery imaged with optical coherence tomography. Invest Ophthalmol Vis Sci. 2012;53: 5819-26.

26. El-Dairi MA, Holgado S, Asrani SG, Enyedi LB, Freedman SF. Correlation between optical coherence tomography and glaucomatous optic nerve head damage in children. Br J Ophthalmol. 2009;93:1325-30.

27. Yanni SE, Wang J, Cheng CS, et al. Normative reference ranges for the retinal nerve fiber layer, macula, and retinal layer thicknesses in children. Am J Ophthalmol. 2013;155:354-60.

28. Kim TW, Kim M, Weinreb RN, Woo SJ, Park KH, Hwang JM. Optic disc change with incipient myopia of childhood. Ophthalmology 2012;119:21-26 e1-3. 